\title{
Simple and Rapid Method for Rat Estrous Cycle Identification Using Crystal Violet- Hormonal Consideration
}

\author{
Nazia Begum ${ }^{1,2^{*}}$, Kandavalli Manipriya ${ }^{2}$, Rahathunnisa Begum ${ }^{2}$, Dr. Veeresh $\mathbf{B}^{3}$ \\ ${ }^{1}$ Research Scholar, University College of Technology, Osmania University, Hyderabad, Telangana, India. \\ ${ }^{2}$ Department of Pharmacology, G. Pulla Reddy College of Pharmacy, Hyderabad, Telangana, India. \\ ${ }^{3}$ Professor, Department of Pharmacology, G. Pulla Reddy College of Pharmacy, Hyderabad, Telangana, India.
}

\begin{abstract}
Rat estrous cycle determination or vaginal smear staining is paramount for studies related to endocrinology and reproduction; in the present study; we have reported a simple and rapid method for estrous cycle determination in rats using crystal violet. With this technique, the identification of stages can be done even on the next day. Hormonal variation in blood and histomorphological changes in ovaries at different stages of the estrous cycle were studied in female Wistar rats, which can be used to determine the hormone levels in works related to hormonal drugs, further ovarian morphology can be used to study changes in ovaries during the estrous cycle. This study aims to report a rapid and simple method for vaginal cytology using crystal violet and to report normal hormonal levels and histomorphology of ovaries in various phases of the estrous cycle.

Key words: Estrous cycle; Vaginal smear; Staining technique; Crystal violet; Sex hormones.
\end{abstract}

\section{INTRODUCTION}

Determination of rat estrous cycle is the most important for the works related to reproductive, endocrinological studies, studies related to hormonal drugs etc. ${ }^{1-3}$ Rat estrous cycle consists of 4 phases: proestrus, estrus, metestrus, and diestrus, that can be identified using vaginal smear, which is considered as a mirror of ovarian function that reflects the activity of sex hormones. The main difference between rodent estrous cycle and the human menstrual cycle is the overall time for a full cycle, the peaks of sex hormones are typically separated in humans whereas they overlap in rodents. ${ }^{4}$ Proestrus and estrus occur in the follicular phase of the ovarian cycle with ovulation taking place towards the end of estrus. Metestrus and diestrus or pseudopregnancy comprise the luteal phase of the cycle. These phases, therefore, correspond to the follicular or proliferative phase and the luteal phase of the menstrual cycle in humans. ${ }^{5}$ Also rodents are more susceptible to estrous cycle disruption from light zeitgebers than are humans. ${ }^{6}$
Though there are many techniques already available for identification and determination of estrous cycle and hormonal consideration, there is always a requirement for better technique than older. Thus, study aims to report a rapid and simple method for vaginal cytology using crystal violet and to report normal hormonal levels and histomorphology of ovaries in various phases of estrous cycle.

\section{MATERIAL AND METHODS}

\subsection{Requirements}

Crystal Violet solution was procured from S.D. fine chemicals, Mumbai. Saline, cotton buds, and methanol obtained from standard sources. Hormones were assayed by chemiluminescence method using instrument ADVIA centaur XP system, Siemens Healthcare Diagnostics Inc. USA. Progesterone was estimated using ADVIA centaur progesterone (PRGE) kit, testosterone using ADVIA centaur Testosterone (TSTO) kit, estrogen using ADVIA centaur E2-6 KIT.

\section{Corresponding author}

Nazia Begum

Email : naziabegum2003@gmail.com 


\subsection{Experimental Animals}

In the present study, 24 female Wistar rats of 7-8 weeks old weighing 130-150g were used. The animals were housed in standard cages in controlled temperature $25^{\circ} \mathrm{C}$, with a $12 \mathrm{~h}$ light and $12 \mathrm{~h}$ dark cycle. Standard laboratory chow and tap water were available ad libitum. After one week of acclimatization period, they were randomly selected for experimental studies. All the experimental procedures were carried out in accordance with committee for the purpose of Control and Supervision of Experiments on Animals guidelines. The study was reviewed and approved by the Institutional Animal Ethics Committee (GPRCP/IAEC/23/19/02/PCL/AE-6A-rats-F-30), G. Pulla Reddy College of Pharmacy, Hyderabad, Telangana, India.

\subsection{Vaginal Smear Collection and Staining Using Crystal Violet}

The vaginal smears were collected using a cotton bud dipped in normal saline (Figure 1A) and air dried (Figure 1B), after which methanol was added to fix it and dried again. Crystal violet was added drop wise to the smears and kept aside for 3 minutes and the slide were washed in running water (Figure 1C) and observed under light microscope without glycerin (Figure 2). After collection and drying of vaginal smears, direct crystal violet can also be added. But perfect identification of the phase is difficult and hence, addition of methanol is an important step for clear smear observation.

In the present technique air drying and methanol, fixation causes double fixation of smear on slide, addition of crystal violet makes the cells colored and easily visible in the microscope, also avoiding fixation with glycerol helps in prevention of fading of the color due to crystal violet and glycerine reaction. Smears fixed and stained
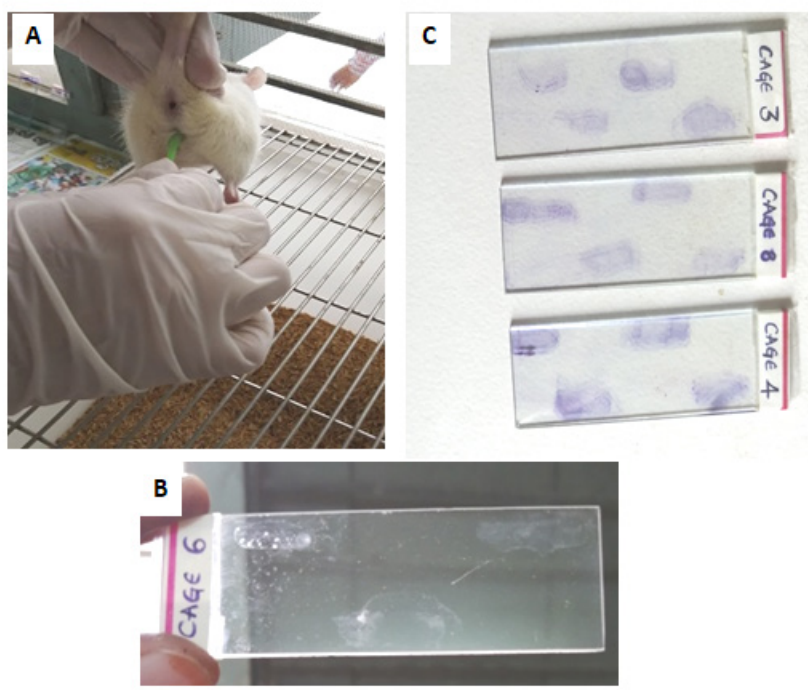

Figure. 1: A: Collection of Vaginal Smear, B: Collection of Smear on Slide, C: Slides after Washing the Stain with this technique will be very useful for research studies, in which smears has to be collected daily with a large number of animals.

\subsection{Hormonal Levels in Various Phases of Estrous Cycle}

Rat hormonal levels are important for endocrinological and metabolic studies. As rats have concise reproductive cycle with overlapping peaks of hormones, one should have good knowledge on the levels of various sex hormones at different phases of the estrous cycle. After collection and identification of estrous cycle, blood samples were collected under isoflurane anesthesia through retroorbital route for various hormonal estimations such as estradiol, progesterone and testosterone.

\subsection{Histomorphology of Normal Ovaries at Various Stages of Estrous Cycle}

Histoarchitecture of ovaries can be crucial for reproductive organ studies and their toxicity. As rats have different morphology of ovaries at different estrous cycle, one should know when to collect the ovaries for histology. After identification of the estrous cycle, the animals were sacrificed, and ovaries were excised. After excision, ovaries were freed from fat, blood and cleaned with ice-cold saline, fixed with $10 \%$ formalin, and analyzed microscopically by staining with hematoxylin and eosin.

\section{CRITICAL PARAMETERS AND TROUBLESHOOTING}

- For mice holding the mice in hand and collection of smear can be preferable, but for rats placing the rat on the cage lid and lifting the tail for collection of smear could be preferable than holding in hand.

- Identification of cell types is easier with $40 x$ objective lens, but identifying a particular phase proportion of each type of cells is important, which can be done with a $10 x$ objective lens.

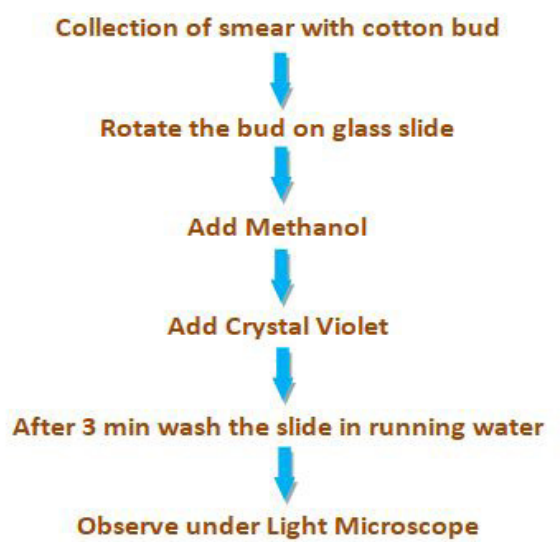

Figure. 2: Collection and Staining of Vaginal Smear 
- As rats have very short reproductive cycle, much time should not be taken between identification of phase and collection of a blood sample for hormonal estimation or ovaries for histology.

\section{RESULTS}

\subsection{Vaginal Smear Collection and Staining Using Crystal Violet}

The images of crystal violet stained slides showing different phases of estrous cycle with original magnification
10X and 40X objective lens are shown in Figure 3. Proestrus smear shows nucleated epithelial cells (Figure 3A and $3 \mathrm{~B}$ ), Estrus smear has cornified cells (Figure $3 \mathrm{C}$ and 3D), metestrus smear consists of leukocytes and cornified cells (Figure 3E and 3F), diestrus phase shows many leukocytes (Figure 3G and 3H). Transition between the two phases can also be easily identified with crystal violet. Figure 4 represents various transition phases under 10x objective lens. Figure 4A shows nucleated cells that are fully matured and show cornification along with nucleus. This is a transition between proestrus to estrous phase.

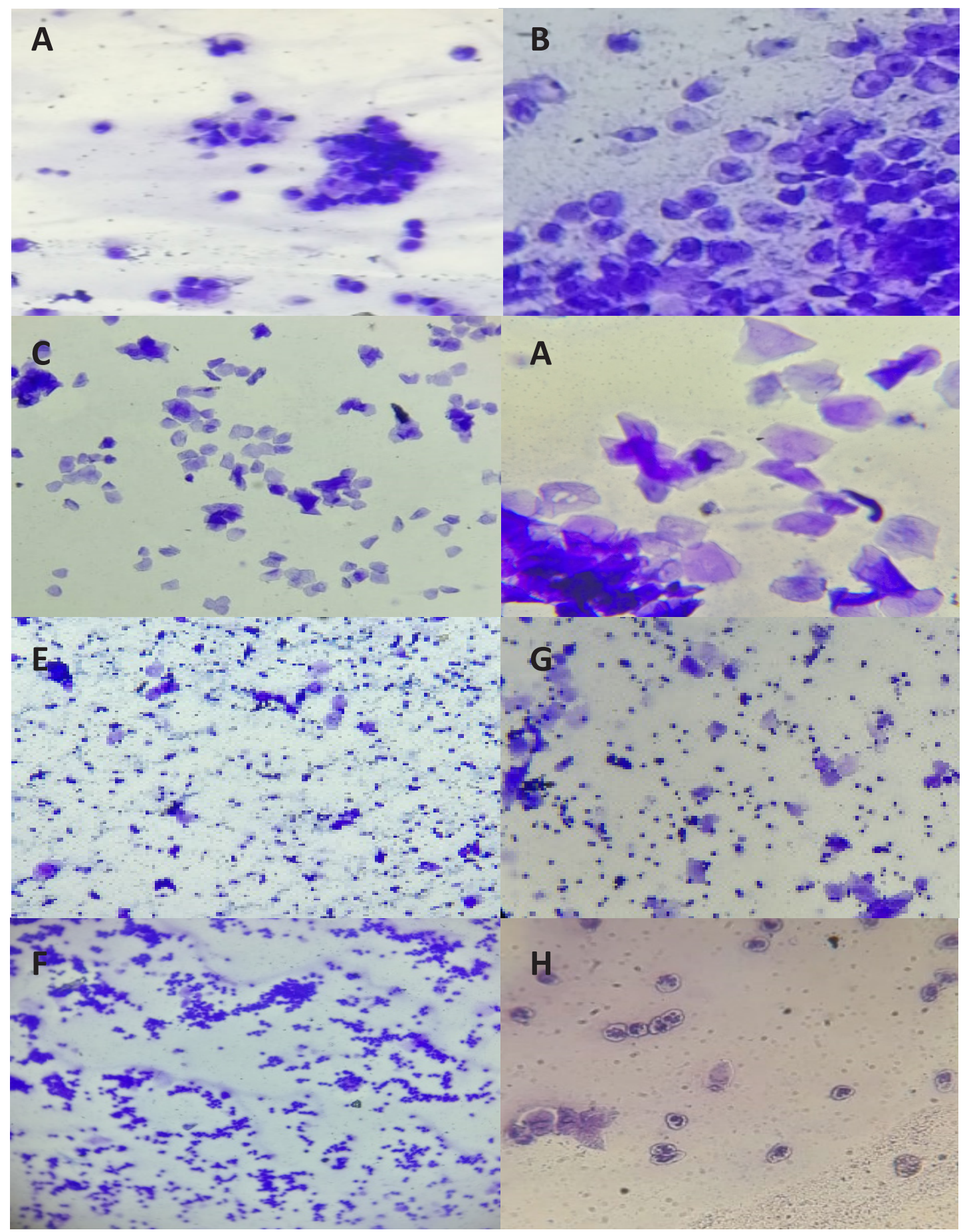

Figure. 3: Vaginal Smears stained with Crystal Violet showing different phases of Estrous cycle: A: Proestrus 10X; B: Proestrus 40X; C: Estrous 10X; D: Estrous 40X; E: Metestrus 10X; F: Metestros 40X; G: Diestrus 10X; H: Diestrus 40X 


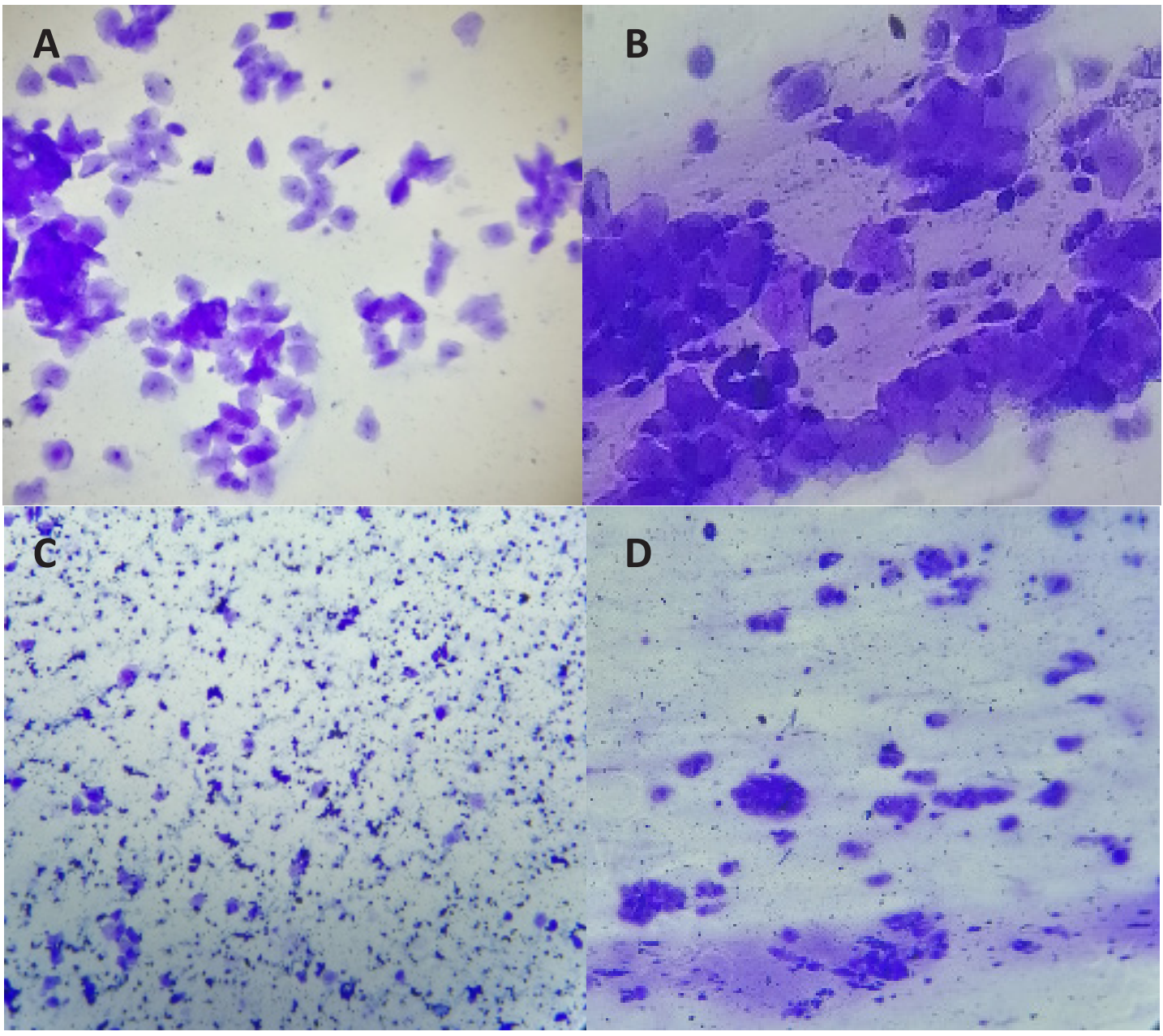

Figure. 4: Phase transition between different Phases A: proestrus to estrus, B: estrus to metestrus, C: metestrus to diestrus, D: diestrus to proestrus, $10 x$ Objective lens

Figure $4 \mathrm{~B}$ has large number of cornified cells that are accompanied with few leukocytes and few nucleated cells; this is a transition between estrous phase to the metestrus phase. Figure $4 \mathrm{C}$ consists of very few cornified cells that are accompanied by many leukocytes; this shows transition between metestrus to diestrus. Figure $4 \mathrm{D}$ shows few leukocytes and few nucleated cells which is transition phase between the diestrus phase and the proestrus. And this is the phase were testosterone levels will be highest.

\subsection{Normal Hormonal Levels in Various Phases of Estrous Cycle}

Plasma Estradiol concentration is low in metestrus and starts elevating at the diestrus phase, further estradiol levels elevate at the proestrus phase, which reaches its maximum level at the evening of proestrus phase and start falling at estrous phase (Figure. 5). Progesterone levels are lowest at the diestrus phase and start increasing at proestrus and reach peak at proestrus evening. Progesterone helps maintain the pregnancy and remains elevated and if pregnancy does not occur, the levels drop at estrous phase and reach lowest at diestrous reaches (Figure 6). Serum testosterone levels starts elevating from metestrous to diestrus and reaches its maximum peak

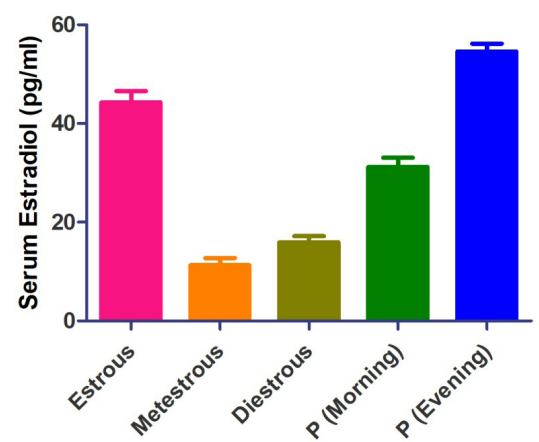

Figure. 5: Serum Estradiol levels during different stages of Estrous Cycle

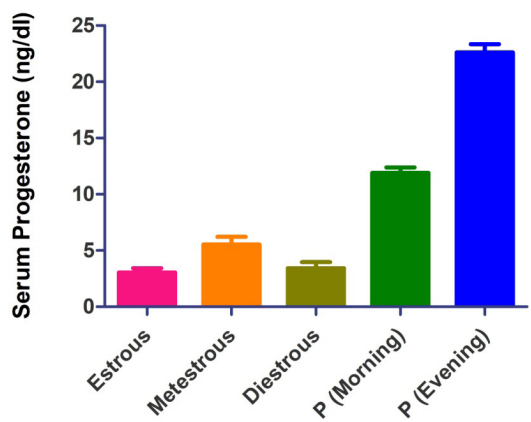

Figure. 6: Serum progesterone levels during different stages of estrous cycle 
at start of proestrus and as the proestrus phase continues the serum testosterone levels declines and further declines at the estrus phase (Figure 7).

\subsection{Histomorphology of Normal Ovaries at Various Stages of Estrous Cycle}

According to the results of histopathology of ovaries at various stages of the estrous cycle (Figure 8), proestrus

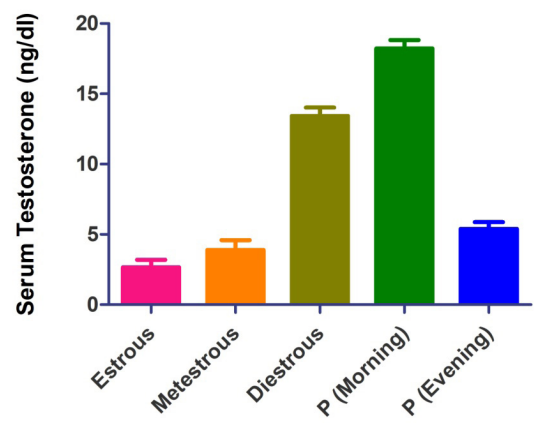

Figure. 7: Serum testosterone levels during different stages of estrous cycle

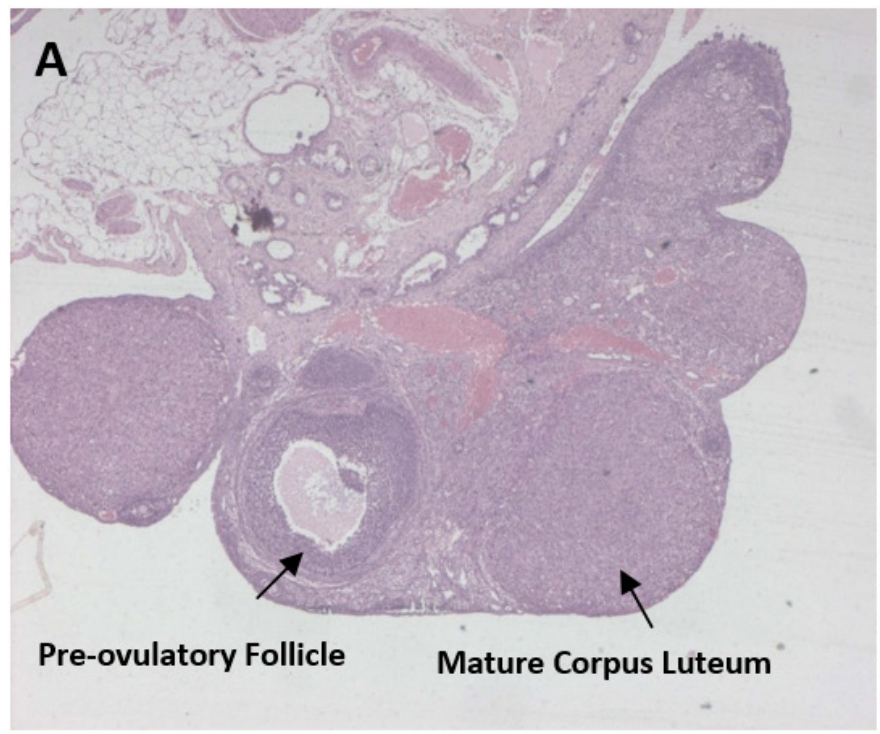

C
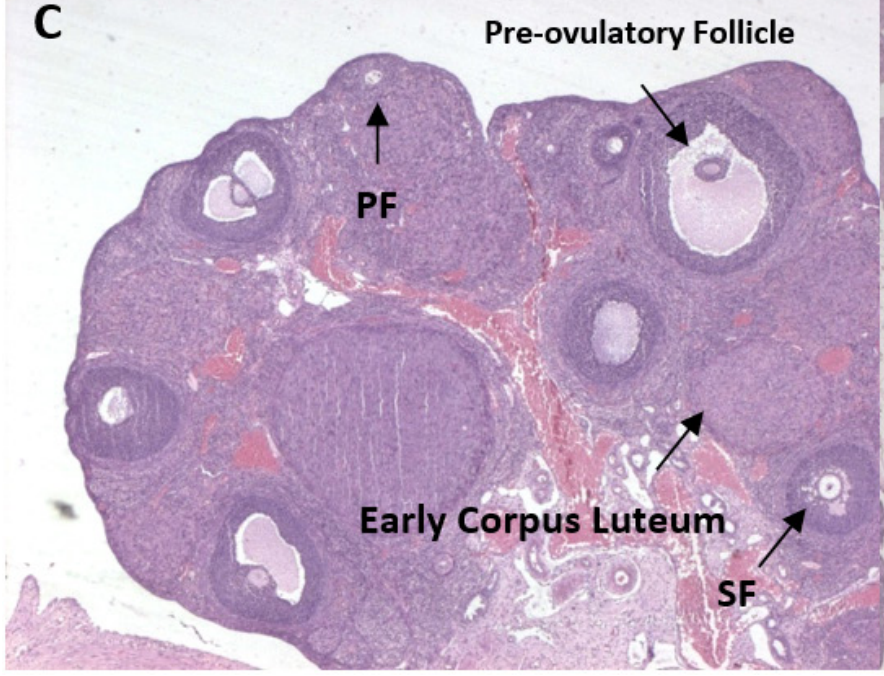

Figure 8: Histoarchitechture of Ovaries of rat at different phases of Estrous Cycle (10x) and diestrus phases have shown mature corpus luteum along with pre-ovulatory follicle. Estrus phase have shown many primary and secondary follicle along with early corpus luteum, and metestrus phase have shown pre-ovulatory and secondary follicles along with early corpus lutem.

\section{DISCUSSION}

Exact identification of rat vaginal smear, hormonal estimation is challenging because of the short cycle of the rat and overlapping hormonal levels. Thus a technique which is rapid and simple would be most beneficial. Previously Marcondes et al., 2012 have shown that vaginal smears can be collected by lavage method and observed directly without any staining techniques, but lavage method and observing without any stain itself is a challenge for a new observer/researcher. ${ }^{7}$ Also McLean et al., 2012 have shown that smears can be collected by lavage method, stained with crystal violet and fixed with glycerol, but we found some of the techniques in this
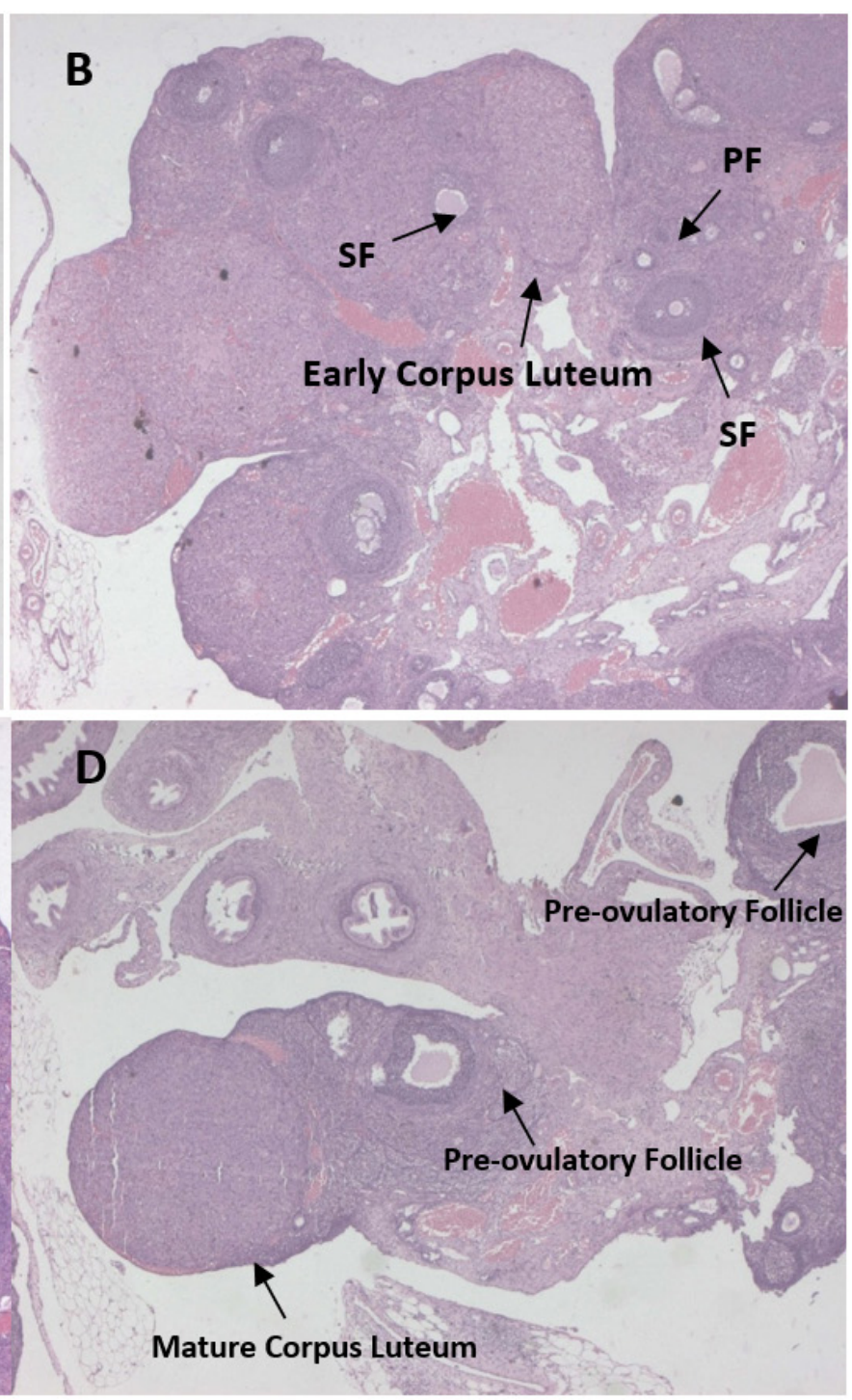

Mature Corpus Luteum 
process require more expertise such as vaginal lavage and slides visualization is not that clear with direct crystal violet staining as that for methanol fixation and then crystal violet staining, also fixing with glycerol can fade crystal violet. ${ }^{8}$ Srinivasan et al., 2017 have shown the crystal violet staining procedure in which the slide has to be dried for 3-4 hours after smear collection prior to crystal violet staining or PAP staining technique, which is a time-consuming step. ${ }^{9}$

In the present work, staining time, on the whole, is of a maximum of 5 minutes, thus overcoming the drawbacks of both above studies; the present work has shown a better and very simplified way for vaginal smear identification. This method takes almost 5-6 minutes for fixing, staining and observation of 4-6 samples.

Estradiol levels in the present study were observed to be high at proestrus evening and start falling at estrus, the concentration was lowest at the metestrus phase. These results are in confirmation with the previous study. ${ }^{10,11}$ Whereas Zenclussen et al., 2014 has shown that estradiol levels are at base at estrous phase. ${ }^{12}$ In the present work, serum progesterone levels were highest at the proestrous evening and lowest at estrous and diestrus phases. This is in confirmation with Stanley \& Scharfman, 2005 and Haim et al., 2003. ${ }^{13}$ In contrast to the work done by Zenclussen et al., 2014 has shown progesterone levels peak at estrous phase. Serum testosterone levels were high at the end of diestrus phase and at the start of proestrus phase, and the levels were lowest at esrous phase. These results are in confirmation with previous work of Haim et al., 2003 and Rush et al., 1982. ${ }^{14}$

\section{CONCLUSION}

The present study developed a simple, rapid and accurate method for identification of estrous cycle in rats. The developed method also provides precise determination of histomorphology and various hormonal levels during different phases of estrous cycle.

\section{ACKNOWLEDGEMENTS}

Authors acknowledge the support received from Principal and staff of G. Pulla Reddy College of Pharmacy.

\section{REFERENCES}

1. Ekambaram G, Sampath SK, Joseph LD. Comparative study on the estimation of estrous cycle in mice by visual and vaginal lavage method. J Clin Diagn Res. 2017;11: AC05-07.
2. Koebele SV, Bimonte-Nelson HA. Modeling menopause: The utility of rodents in translational behavioural endocrinology research. Maturitas. 2016;87: 5-17.

3. Xu T, Li X, Yang L, Zhang Y, Zhang L et al. Impact of cold exposure on the reproductive function in female rats. Biomed Res Int. 2018;3674906(6).

4. Paccola CC, Resende CG, Stumpp T, Miraglia SM, Cipriano I. The rat estrous cycle revisited: a quantitative and qualitative analysis. Anim. Reprod. 2003;10: 677-683.

5. Sato J, Nasu M, Tsuchitani M. Comparative histopathology of the estrous or menstrual cycle in laboratory animals. Journal of Toxicologic Pathology. 2016;29: 155-162.

6. Goldstein CA, Smith YR. Sleep, circadian rhythms, and fertility. Current Sleep Medicine Report. 2016;2: 206-217.

7. Marcondes FK, Bianchi FJ, Tanno AP. Determination of the estrous cycle phases of rats: Some helpful considerations. Braz. J. Biol. 2002;62: 609-614.

8. McLean AC, Valenzuela NS, Fai S, Bennett SA. Performing Vaginal Lavage, Crystal Violet Staining, and Vaginal Cytological Evaluation for Mouse Estrous Cycle Staging Identification. J Vis Exp. 2012;15: e4389

9. Srinivasan MR, Sabarinathan A, Geetha A, Shalini K, Sowmiya M. A comparative study on staining techniques for vaginal exfoliative cytology of rat.Journal of Pharmacology and Clinical Research. 2017;3: 1-5.

10. Caligioni CS. Assessing reproductive status/stages in mice. Current protocols in neuroscience. 2009;Appendix-4I.

11. Haim S, Shakar G, Rossene E, Taylor AN, S Ben-Eliyahu. Serum levels of sex hormones and corticosteronethroughlot 4- and 5-day estrous cycles in Fischer 344 rats and their simulation in ovariectomized females. J. Endocrinol Invest. 2003;26: 1013-1022.

12. Zenclussen ML, Casalis PA, Jensen F, Woidacki K, Zenclussen AC. Hormonal Fluctuations during the Estrous Cycle Modulate Heme Oxygenase-1 Expression in the Uterus. Frontiers in endocrinology. 2014;5: 32.

13. Stanley K, Scharfman H. A woman's prerogative.Nature Neuroscience. 2005;8: 697-699.

14. Rush ME, Blake CA. Serum Testosterone concentrations during the 4-day estrous cycle in normal and adrenalectomized rats (41334). Proceedings of the society for experimental biology and medicine. 1982;169: 216-221.

How to cite this article: Begum N, Manipriya K, Begum R, Veeresh B. Simple and Rapid Method for Rat Reproductive Stage Identification Using Crystal Violet- Hormonal Consideration. International Journal of Applied Pharmaceutical Sciences and Research. 2020;5(4):54-59. doi: https:// doi.org/10.21477/ijapsr.5.4.1

Source of Support: Nil.

Conflict of Support: None. 